\title{
NUMERICAL MODELING OF ECCENTRIC JET PRECOMBUSTOR
}

\author{
Sun Yezhu \\ Huaneng Power Intl. Inc. \\ Institute of Mechanics, Chinese Academy of Sciences, \\ P.R. China, Beijing. \\ Yezhu_Sun@hotmail.com
}

\section{ABSTRACT}

This thesis presents a numerical modeling on the cold flow field of the eccentric jet precombustor developed by the Institute of Mechanics, Chinese Academy of Sciences.

The numerical modeling is to research the flow field structure of the eccentric jet precombustor, to study the factors which affect the recirculating region produced in the precombustor by eccentric jets and their affecting rules, then the results of the study will play the guiding and referenced roles in the optimizing design and operation of the eccentric jet precombustors.

The effects of precombustor structure and operating parameters on flow field characteristics and possible effects on combustion stability in these two types of eccentric jet precombustor are numerically modeled, a detailed numerical analysis on the effects of eccentric jet precombustor's structure include the shape of eccentric jet precombustor, the length of precombustor, the position, area, obliquity and shape of primary jet (main jet near center), the position and area of secondary jet (auxiliary jet near the wall), the momentum rate of primary jet to secondary jet etc. The code is used to solve the velocities fields, turbulent kinetic energy fields and other parameters. On this basis a comparison is made between the investigated cases and the most effective conditions are determined.

\section{INTRODUCTION}

The eccentric jet precombustor is a kind of burner, which possesses the function of burning and stabilizing flame in the precombustor, used in the industrial boiler. The mechanism of the eccentric jet precombustor is that two asymmetric jet go into the confined space with cylindrical or rectangular section, the inducing function of two jets induce the fluid around the jets to form a big recirculating region in the confined space, the back fluid may brings burning mixtures of fuel and air with heat into the recirculating region, and heats and burns the new mixtures of fuel and air coming with jets, so the precombustor can act as the stabilizing flame and burning without the igniting oil gun.
The paper deals with the forming and operation rule of the recirculating region of the eccentric jet precombustor, and is a part of the project of National Natural Science Fund of China, "the research on the character of emission, the mechanism and rule of stabilizing flame of asymmetric jet precombustor".

There are several factors influencing the recirculating region of the eccentric jet precombustor but the inducing of the jet is the main factor preconditioning the flow field character thus influencing the form and the size of recirculating region. An assessment of the jet's inducing influence on the form of recirculating region requires a detailed study of the process. Computer simulation of the flow field character is an effective tool for such a study as it allows us to obtain any detailed information on the main process characteristics.

The present investigation deals with the industrial application of the eccentric jet precombustor originally designed for igniting. The aim of the paper is to investigate numerically the influence of the jets configuration and parameters on the recirculating region.

\section{NOMENCLATURE}

Eccentric jet precombustor, flow field structure, recirculating region, combustion stability, turbulent model.

\section{COMPUTATIONAL DETAILS}

The software is based on Favre averaged 3D isothermal transport equations, the standard k-e turbulence model. To study two different sections of eccentric jet precombustors, the cylindrical coordinate is employed for circular section and Car! coordinate for rectangular section. The numerical modeling codes are based on three dimensional SIMPLE (Semi-Implicit Methods for Pressure Linked Equation) algorithm which was developed by S.V. Patankar and D.B. Spalding, the finite difference method is employed to divide the geometry of eccentric jet precombustors into the control volumes with nonuniform grids. Along the direction of jets is $x$ direction (I direction); the vertical direction is y direction(J direction), the 
horizontal direction is $\mathrm{z}$ direction( $\mathrm{k}$ direction) for Carl coordinate; the radial direction is $y$ direction( $J$ direction), the tangential direction is $\mathrm{z}$ direction( $\mathrm{k}$ direction) for cylindrical coordinate. Because of the center symmetry of precombustor, the half of the section of the precombustor is modeling numerically, the computational grid composed $20 \times 23 \times 13$ control volumes for the precombustor with rectangular section, $21 \times 13 \times 13$ control volumes for circular section.

\section{PRECOMBUSTOR GEOMETRIES}

The investigated precombustor dimensions are $450 \mathrm{~mm} \times 1200 \mathrm{~mm}$ (diameter $\mathrm{x}$ length) for the circular section, $360 \mathrm{~mm} \times 230 \mathrm{~mm} \times 720 \mathrm{~mm}$ (height $\mathrm{x}$ width $\mathrm{x}$ length). The flow velocity of main jet is evenly about $25 \mathrm{~m} / \mathrm{s}$, the velocity of secondary jet is different from 0 to $60 \mathrm{~m} / \mathrm{s}$. for the precombustor with rectangular section, the main jet's inlet dimensions are $90 \mathrm{~mm} \times 160 \mathrm{~mm}$, located about $60 \mathrm{~mm}$ below the center, the secondary jet inlet is very thin and near bottom wall; for the precombustor with circular section, the main jet inlet's radius is $1 / 4$ of the radius of the section, and one radius from the center of the section, the secondary jet also is a thin jet near the bottom wall.

\section{RESULTS AND DISCUSSIONS}

The flow field structure of the precombustor with circular section is basically same as the precombustor with rectangular section. The flow fields are shown in Figure 1, and Figure 2. for the conjunct effects of two jets, we call the big jet main jet, the jet near the wall secondary jet, there is a big recirculating region produced in the upper part of precombustor.

In the flow filed vector chart, the direction of arrowhead denotes the flow direction, the end of the arrow denotes the place, and the length of arrow denotes the projection of dimension of velocity on the plane.

Figure 2, when the main jet goes into the precombustor, as the inducing effects of the secondary jet, the flow direction lean to the bottom wall, then go straightly. The upper part fluids over the main jet are induced downward, the upper space is filled by the backward fluid, so as these flows produce recirculating region in the upper part of precombustor.

The flow field of precombustors with different section. The flow field of precombustor with rectangular section is shown Figure 2, there is a vortex between the secondary jet and bottom wall, it is small recirculating region, but the velocity is quite big because of the strong inducing effect of the secondary jet and the space is very small, there are no enough fluid induced, so make a very high negative pressure region, there is very strong inducing effect and inducing velocity is very big. For the precombustor with circular section, there is space beside the secondary jet, the fluid can flow into the small space from the two sides, and then it cannot form a very small vortex with strong inducing effect.
In Figure 2, there are three flow field vector charts of $k=1,3,5$ section, the flow filed structure are very similar, the similar images show that the flow field characters two dimension, the analysis of the reason is the confined space characters two dimension and the jet inlet especially the secondary jet inlet characters two dimension. But the character of two dimension is not so obvious for the precombustor with circular section, there is enough three dimension character, shown in Figure 3, $I=3,6,13,15$ sections, there is already secondary flow in the section $I=13$. these characters show it characters three dimension.

The comparison of the precombustors with different length. Figure 4 shows the flow field of precombustors with different length, the flow images of precombustors with different length are similar at the same length part, only at the outlet of short precombustor there small difference from the long precombustor, because of the effect of the outlet, and the back flow quantity is same too, it shows the numerical modeling software is correct and the result is effective. It makes the designers to select the different length precombustor according to the boiler's size.

The effect of location of main jet inlet on the flow filed of precombustor. Figure 5 shows the flow field of different location of the main jet inlet, up, middle, down, three locations, the height difference of them is half of the height of inlet. The flow field structures are similar and only the inlet flows are different. Figure 6 shows the quantity of backward flow curves of three inlet locations of main jet, the quantity are same too, it shows that the change of inlet location of main jet makes very small change of flow field and backward flow quantity under the condition of the secondary jet inducing effect.

The main jet inlet's area and shape has some effect on the flow field of precombustor. In Figure 7 , we can see the difference between the different areas, $S a: S b=2: 1$. the inlet shape of the Sb is more flat than the inlet of Sa when the other conditions are same. Because the inlet becomes flat, the main jet flow to the bottom wall more quick, the flow field has some different at fore part of the precombustor, but when it flow to middle, the flow field become same, and the quantity of recirculating flow rate increases. The analysis is that because the inlet of main jet becomes flat, the jet's rigidity becomes weak, it is easy to be induced downward by the secondary jet, and it bends easily. And the inlet's area decreases, the quantity of fluid into the precombustor decreases, so the recirculating flow rate increases relatively.

The oblique angle of the main jet effect on the flow field of precombustor is not so big when there is the inducing effect of the secondary jet. In Figure 8, the flow field of precombustors has some difference when the inlet angle of main jet is different, the character of the flow field has no difference. When the inlet of main jet is canted 12 deg upward, the main jet is not induced 
quickly, and induced gradually, as the inlet is not canted upward, the main jet is induced directly. But the flow field structure has no intrinsically differences when there is the inducing effect of the secondary jet. If there is not the secondary jet, the flow field change largely, it is difficult to form the recirculating region with the oblique angle under this condition. In fact, if there is oblique angle, the main jet includes air and fuel(coal), the inertia of the coal is bigger, then it can go into the center of recirculating region directly, and heated to burn. The next investigation will prove the idea.

The comparison of recirculating rate changes with the different momentum rate changes. Figure 9 shows the recirculating rate curve at different momentum rate, when other conditions are definite, the maximum recirculating increases with the momentum rate increases, at begin it increases more quickly, and the increasing velocity become slow. It show that the inducing effect of the secondary jet increases quickly with the momentum rate increases at begin, afterward the inducing effect is restricted by the precombustor's space, then the increasing velocity of the maximum recirculating rate becomes slow. At the same time, the center of recirculating region move downward with the momentum rate increases, the recirculating region become big from small, and the recirculating region becomes unclose from the close. It shows that when we need a recirculating region no matter close or unclose, we may operate the secondary jet's parameter to make a required recirculating region.

It must be stressed that the inducing effect of the secondary jet play main pole to produce the recirculating region, and changing parameter of the secondary jet may control the dimension of the recirculating region, if without the inducing effect of the secondary jet, it is difficult to obtain a appropriate recirculating region.

\section{CONCLUSIONS}

The results show that the shape of precombustor, the structure and operating parameters of the primary and secondary jets all have effects on the flow field structure. Their combined action can produce a large recirculating region, which is the main source for flame stability in the eccentric jet precombustor. Within precombustor with definite length, two jets' effect reciprocally can make a large recirculating region; if without the secondary jet, the recirculating region is difficult to form, the secondary jet play inducing role; the recirculating regions are different in the precombustors with different section, the recirculating region of precombustor with the circular section possesses three dimensional characteristic, the recirculating region of precombustor with the rectangular section does not possess obvious three dimensional characteristic; with the increase of velocity of secondary jet, the intensity of recirculating region will increase while the velocity of main jet is definite, and the center of the recirculating region will move downstream; the shape, size and area of the main jet possess definite effects on the flow field structure, the input angle of main jet possess less effects on the recirculating region, but if without the secondary jet, the input angle of the main jet will play important role on flow field structure, and the recirculating region is difficult to form; the maximum recirculating quantity has a definite relationship with the momentum rate of main jet and secondary jet.

A further improvement of the flow properties within the precombustor and stabilizing flame could be supported and reached by detailed investigation of precombustor processes under various conditions such as jets' operating parameters and the rate of fuel and air etc. The CFD codes allow the precombustor designers to test in advance the precombustor behavior under various conditions in order to achieve the optimal design and operation in every specific case.

\section{ACKNOWLEDGMENTS}

The National Natural Science Foundation of China, author graduated from Institute of Mechanics, Chinese Academy of Sciences.

\section{REFERENCES}

1. Zhan huanqing etc., eccentric jet stabilizing flame method and device, patent, 1989.

2. Launder, B.E., and Spalding, D.B., The numerical computation of turbulent flows, Comput. Meth. Appl.

Mech. Eng., 3, pp. 269-289, 1974.

3. Patankar, S.V., and Spalding, D.B., A calculation procedure for heat, mass and momentum transfer in threedimensional parabolic flows, Int. J. Heat Mass Transfer, 15, pp. 1787-1806, 1972.

4. Patankar, S.V., Numerical modeling of heat transfer and flow, Beijing Science Press, 1984.

5. Tao Wenquan, Numerical heat transfer, Press of Xi'an Jiaotong University, 1988.Put references here.

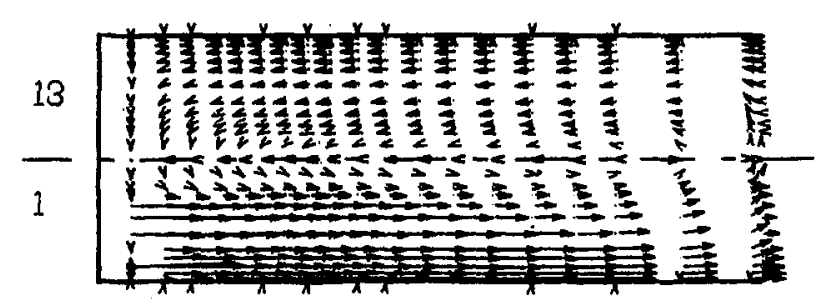

Fig. I flow field vector chart of cylindrical section at $k$ direction (momentum rate $=0.5$ ) 


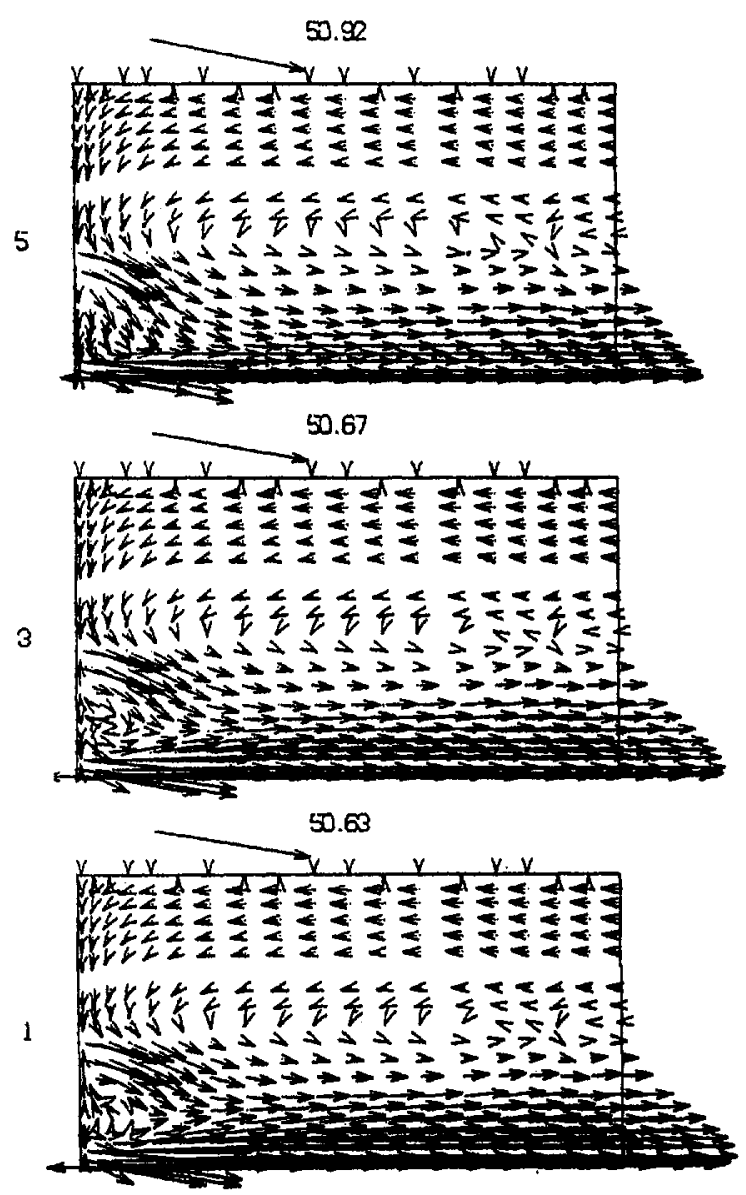

Fig.2 flow field vector chart at different $k$ sections (Momentum rate $=3.63$ )

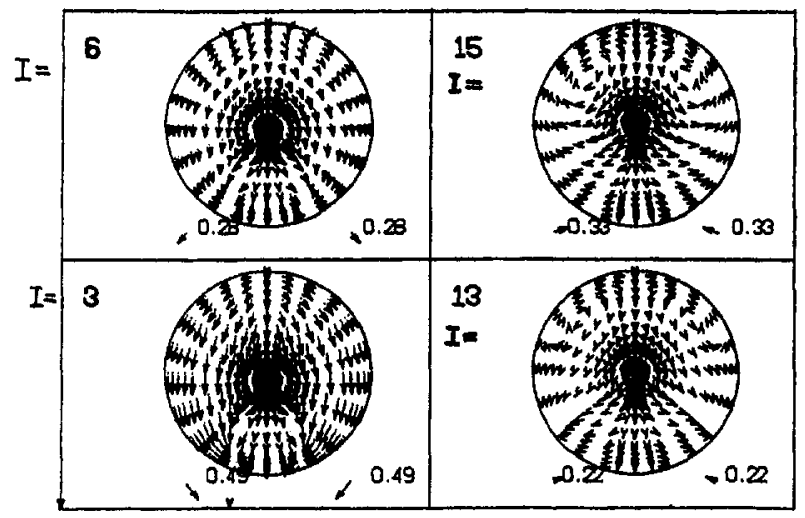

Fig.3 flow field vector chart of different $x$ direction at cylindrical section
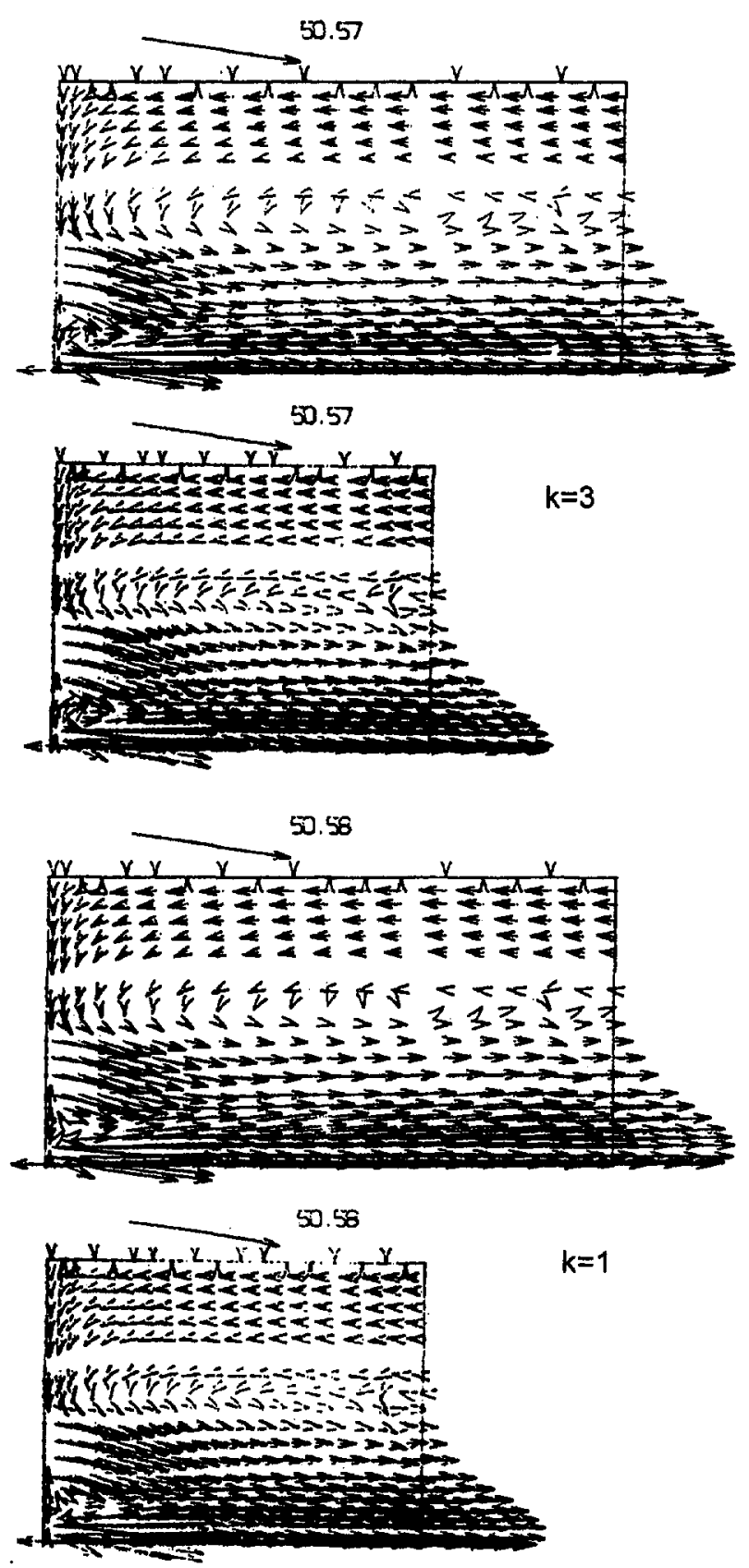

$k=1$

Fig.4 comparison of two different length precombustors $(k=1,3)$ 


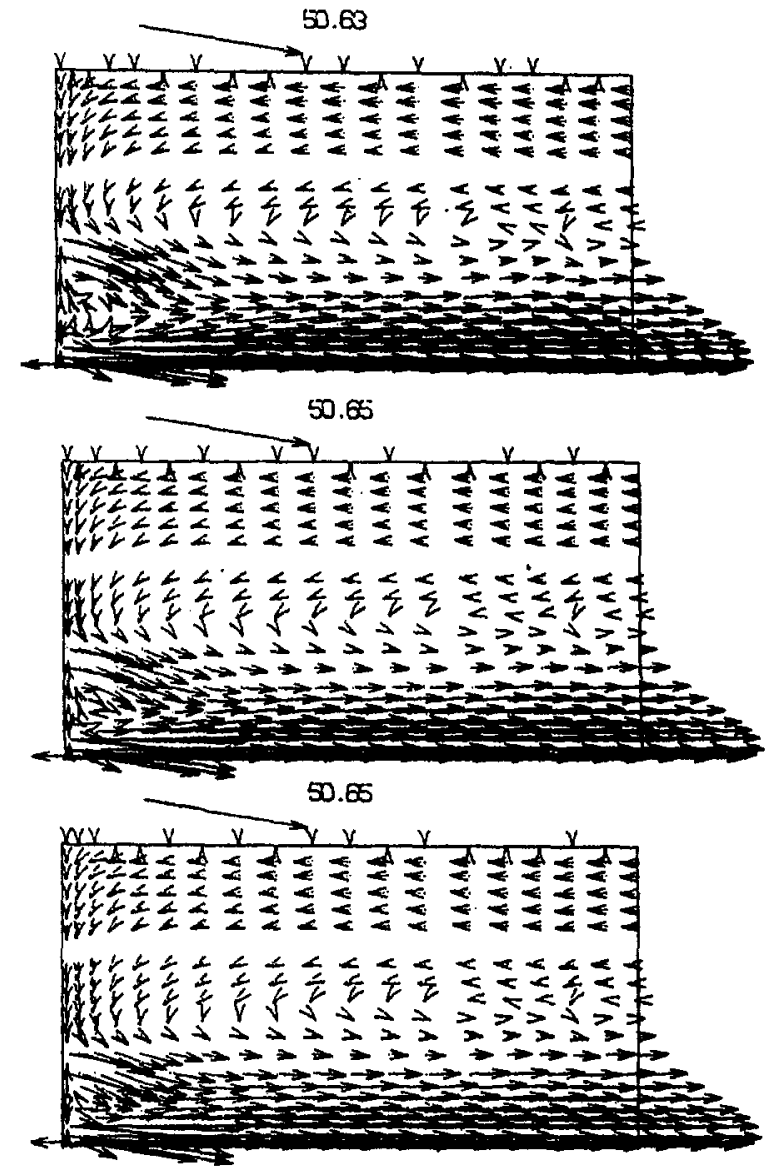

Fig.5 comparison of flow field vector chart at $k=1$ on different location of main jet inlet

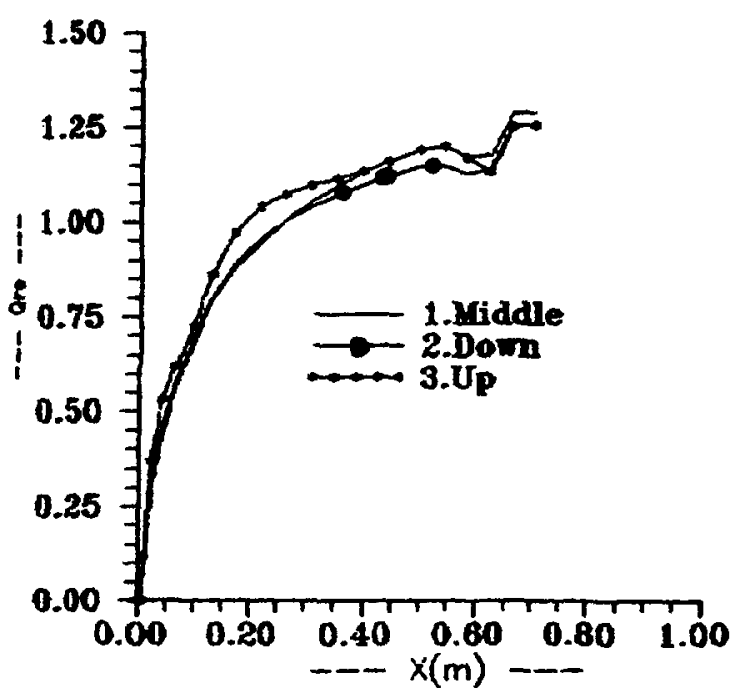

Fig.6 comparison of recirculating rate at different location of main inlet
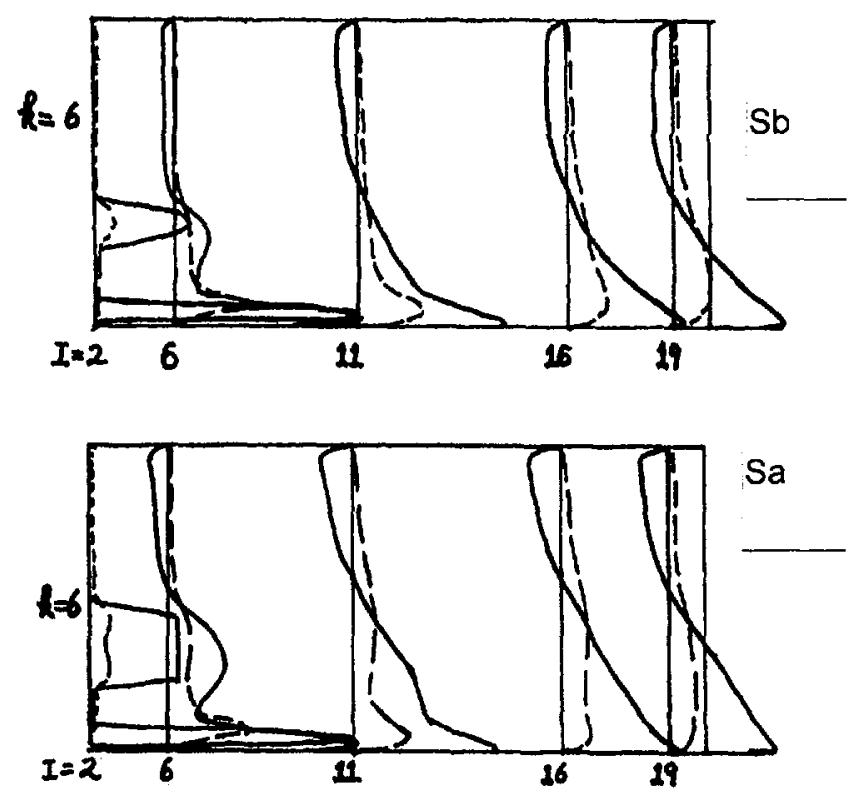

Fig. 7 comparison with the change of area and shape of the main jet inlet $(\mathrm{Sa}: \mathrm{Sb}=2: 1)$

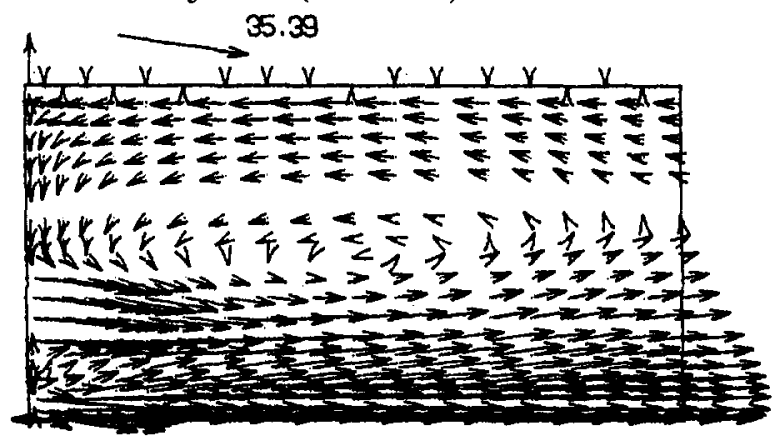

a. $\alpha=0^{\circ}$

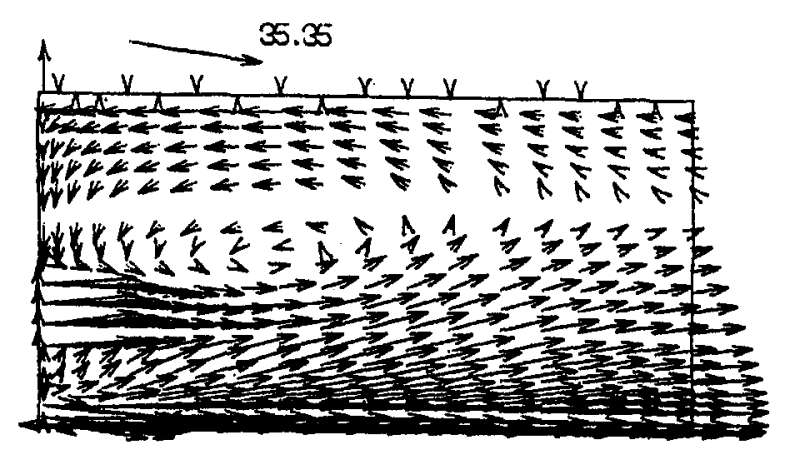

$$
a^{\prime} \alpha=12^{\circ}
$$

Fig.8 Flow field vector chart, main jet with or without oblique angle 


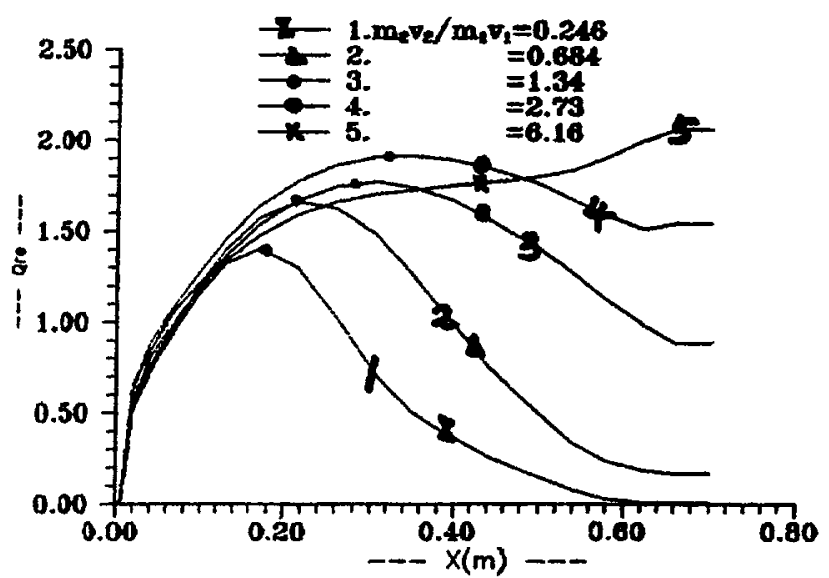

Fig.9 comparison of recirculating rate at different momentum rate 